\title{
Caracterização do desempenho de destreza manual pelo teste caixa e blocos em crianças e adolescentes brasileiros
}

\author{
Manual skill performance by box and blocks test in brazilian children \\ and adolescents
}

\author{
Bruna Paula Boe De Almeida Turco${ }^{1}$, Raquel Cymrot ${ }^{2}$, Silvana Maria Blascovi-Assis ${ }^{3}$
}

http://dx.doi.org/10.11606/issn.2238-6149.v29i2p164-169

Turco BPBA, Cymrot R, Blascovi-Assis SM. Caracterização do desempenho de destreza manual pelo teste caixa e blocos em crianças e adolescentes brasileiros. Rev Ter Ocup Univ São Paulo. 2018 maio-ago.;29(2):164-9.

RESUMO: O teste Caixa e Blocos (TCB) tem se mostrado eficaz para avaliar a destreza manual, que se caracteriza pela capacidade das mãos e dos dedos para desempenhar uma tarefa que requer movimentos coordenados, como a manipulação de objetos sob os aspectos de velocidade, resistência e força. O objetivo desse estudo foi caracterizar o desempenho de destreza manual pelo TCB para a faixa etária de 7 a 14 anos. O estudo contou com 105 participantes, de ambos os sexos, sem apresentação de anormalidades de membro superior, divididos em dois grupos: (7-10 anos) e (11 a 14). Os resultados mostraram melhor desempenho para o lado dominante (direita) no TCB. Em relação à comparação de grupos por idade o grupo de 11-14 anos obteve melhor desempenho, demonstrando que quando maior a idade, melhor o desempenho no teste para ambas as mãos ( $\mathrm{p}=0,000$ para mão dominante e mão não dominante para o sexo feminino e $\mathrm{p}=0,000$ e $\mathrm{p}=0,001$ para mão dominante e mão não dominante no sexo masculino). Pode-se concluir que, para a população estudada com desenvolvimento típico, quanto maior a idade, melhor o desempenho no teste caixa e blocos para ambas as mãos e que, a partir dos dados coletados, foi possível apresentar parâmetros de desempenho para esse teste na faixa etária entre 07 a 14 anos, faixa etária não contemplada na proposta do estudo original de Mathiowetz em 1985.

Descritores: Destreza motora; Mãos; Estudos de avaliação como assunto; Crianças; Adolescentes; Brasil.
Turco BPBA, Cymrot R, Blascovi-Assis SM. Manual skill performance by box and blocks test in brazilian children and adolescents. Rev Ter Ocup Univ São Paulo. 2018 MayAug.;29(2):164-9.

\begin{abstract}
The Box and Blocks Test (BBT) has been shown to be effective in evaluating manual dexterity, which is characterized by the ability of the hands and fingers to perform a task requiring coordinated movements, such as manipulating objects under the speed, force. The aim of this study was to characterize the performance of manual dexterity by BBT for the age group between 7 and 14 years. The study consisted of 105 participants, both sexes, divided into two groups: (7-10 years) and (11-14) without presenting upper limb abnormalities. The results showed better performance for the preferred side (right) in the BBT. Regarding the comparison of groups by age, the group of 11-14 year olds performed better, proving that the greater the age, the test performance for both hands is better $(\mathrm{p}=0,000$ for preferred hand and not preferred hand for the female and $p=0.000$ and $p=$ 0.001 for preferred hand and not preferred hand in males). It can be concluded that, for the study population with typical development, the performance in the test box and blocks was better for the oldest age group for both hands and that, from the data collected, it was possible to present performance parameters for this test in the age group between 07 and 14 years old, age which had not been contemplated in the original study proposal by Mathiowetz in 1985 .
\end{abstract}

Keywords: Motor skills; Hand; Evaluation studies as topic; Child; Adolescent; Brazil.

O artigo é parte da pesquisa desenvolvida no Programa Institucional de Iniciação Científica da Universidade Presbiteriana Mackenzie. O material é parte da pesquisa intitulada "Caracterização do desempenho de destreza manual em crianças, jovens e adultos brasileiros". Apresentado como pôster no IV Congresso Internacional e XXIV Brasileiro da ABENEPI, Rio de Janeiro, em 2017.

Apoio financeiro: MackPesquisa

1. Universidade Presbiteriana Mackenzie, Curso de Fisioterapia, São Paulo, SP. ORCID: https://orcid.org/0000-0001-8502-8624. E-mail: e-mail: bruna. turco@gmail.com.

2. Universidade Presbiteriana Mackenzie, Docente da escola de Engenharia, São Paulo, SP. ORCID: https://orcid.org/0000-0001-9874-4507. E-mail: mail rcymrot@gmail.com.

3. Universidade Presbiteriana Mackenzie, Docente do Curso de Fisioterapia e Programa de Pós-Graduação em Distúrbios do Desenvolvimento, São Paulo, SP. ORCID: http://orcid.org/0000-0002-5437-891X. E-mail: silvanablascovi@gmail.com.

Endereço para correspondência: Silvana Maria Blascovi-Assis. Programa de Pós-Graduação em Distúrbios do Desenvolvimento. Rua da Consolação, 930, prédio 28. São Paulo, SP, Brasil. CEP: 01302-907. 
Turco BPBA, et al. Caracterização do desempenho de destreza manual. Rev Ter Ocup Univ São Paulo. 2018 maio-ago.;29(2):164-9.

\section{INTRODUÇÃO}

Ae possui capacidade de realizar movimentos finos, controle de força e precisão. É utilizada também para a manipulação e preensão de objetos, tornando-se um instrumento importante para o desempenho satisfatório das atividades de vida diária por sua capacidade de executar habilidades de destreza e funções diversas ${ }^{1}$.

A destreza manual é definida como a capacidade das mãos e dos dedos para realizar uma tarefa que exija movimentos coordenados, como a manipulação de objetos sob ação que envolve velocidade, resistência e força. Já a destreza dos dedos, requer o manuseio de objetos pequenos e habilidade para executar dadas manipulações ${ }^{2}$.

Alguns instrumentos de avaliação podem ser usados para quantificar o desempenho da capacidade manual de um indivíduo, como o teste caixa e blocos (TCB) validado e padronizado por Mathiowetz et al. ${ }^{3}$ e validado no Brasil para a população normal, com 446 participantes e para 117 pacientes com esclerose múltipla, ambos os grupos com participantes entre 15 e 86 anos $^{4}$, se mostrando sensível na detecção de mudança na funcionalidade de movimentos do membro superiores também para pessoas com outros diagnósticos ${ }^{5}$.

Considerado um teste simples e de fácil aplicação para averiguar a função manual, o TCB tem como objetivo avaliar e quantificar a destreza manual grossa e habilidades do participante. $\mathrm{O}$ mesmo permite observar a medida de tempo e resistência na realização do teste ${ }^{5}$.

O TCB é constituído de uma caixa de madeira de $53,7 \mathrm{~cm}$ de comprimento, com uma divisória no meio sendo maior que as bordas, a divisória consiste de dois orifícios no meio que vão ajudar apenas no transporte da caixa,150 blocos de madeira com $2,5 \mathrm{~cm}$ de lado, esses blocos são divididos igualmente e pintados das cores primarias ${ }^{3,4}$.

Para aplicação do teste é necessário um ambiente silencioso, no qual o examinando é orientado a sentar-se em uma cadeira que deve ser adequada conforme a sua altura com a caixa posicionada a sua frente horizontalmente sobre uma mesa, que permite total visão da caixa ${ }^{4}$.

$\mathrm{O}$ examinador deve orientar o participante sobre como proceder ao teste. O teste é simples e consiste no transporte dos pequenos cubos de madeira de um lado para o outro durante um minuto. Esses blocos devem ser levados de uma extremidade a outra. No final o número de blocos deve ser registrado para membro superior dominante e não dominante, mediante duas tentativas. $\mathrm{O}$ examinador deve sempre levar em consideração o seu comando verbal para melhor aplicação do teste, estimulando cada vez mais o participante a conseguir realizar maior número de transferências de blocos dentro do tempo estimado ${ }^{4,6}$.

Ao iniciar o teste, o avaliado deve ser instruído que comece com a mão dominante, para isso pode ser aplicado um teste de lateralidade, o qual define se o sujeito é destro ou canhoto can $^{4,7}$.

Esse teste tem sido utilizado no país em diversos estudos, em sua maioria voltados para populações com alterações no desenvolvimento e diagnósticos clínicos neurológicos ou ortopédicos. Estudo com portadores de síndrome de Down entre 7 e 15 anos mostra que não há diferença significante na destreza manual com crianças de 7-9 anos e as de 14-15 anos, indicando que nessa síndrome essas habilidades parecem não evoluir com o avanço da idade, diferentemente da população com desenvolvimento típico ${ }^{5}$. O desempenho motor fino já foi avaliado no Brasil em grupos com síndrome de Down por meio de outros instrumentos padrão ouro, como a Bayley Scales of Infant and Toddler Development-BSITD-III, constatando desempenho inferior ao grupo típico, confirmando os dados encontrados por meio do $\mathrm{TCB}^{8}$.

Alguns estudos vêm demonstrando que a destreza manual pode estar relacionada com o melhor desempenho em algumas tarefas, como a escrita. Para que um indivíduo adquira esta habilidade é necessário que haja coordenação dos movimentos finos e precisos, com uso especializado da musculatura intrínseca da mão ${ }^{9}$. A literatura também refere que pessoas com dificuldades de aprendizagem tendem a apresentar atrasos na coordenação e destreza manual e nas habilidades motoras finas ${ }^{10}$.

Para que a avaliação da destreza seja precisa torna-se importante o uso de instrumentos que possam quantificar o desempenho em tarefas motoras para todas as idades. Mathiowetz et al. ${ }^{3}$ propuseram o TCB com parâmetros para as idades compreendidas entre 20 a 94 anos. No Brasil, o TCB foi utilizado para a faixa etária entre 15 a 86 anos, com grupos típicos e com esclerose múltipla ${ }^{4}$. Guimarães et al. ${ }^{5}$ utilizaram no Brasil o teste para grupos típicos e com Síndrome de Down, contemplando as idades entre 7 a 9 e 14 a 15 anos. Todavia, não foram encontrados estudos com o TCB que padronizem o escore de desempenho para crianças e jovens com menos de 15 anos, sem disfunções de membros superiores, ou seja, sem a presença de quadros clínicos que comprometem suas funções manuais.

Desta forma, torna-se relevante investigar a distribuição média dos escores para o teste nessa faixa etária para a população brasileira em estudos que envolvam grande número de pessoas para que se possam estabelecer parâmetros e estimativas de desempenho em diferentes faixas etárias e possíveis mudanças de desempenho com o avanço da idade. 
Turco BPBA, et al. Caracterização do desempenho de destreza manual. Rev Ter Ocup Univ São Paulo. 2018 maio-ago.;29(2):164-9.

\section{OBJETIVO}

Este estudo teve por objetivo principal, a caracterização do desempenho de destreza manual pelo Teste Caixa e Blocos (TCB) em crianças e adolescentes entre 07 a 14 anos.

\section{PROCEDIMENTOS METODOLÓGICOS}

Foram convidadas a participar 120 pessoas, de ambos os sexos, entre 7 e 14 anos, divididas em dois grupos: Grupo 1 (7-10 anos) e Grupo 2 (11-14 anos), com desenvolvimento típico. Foram critérios de exclusão: possuir algum diagnóstico ortopédico, reumático ou neurológico que pudessem comprometer o desempenho dos membros superiores nas tarefas propostas e apresentar ambidestria pelo teste de preferência manual.

Os dados foram coletados por meio de uma avaliação transversal em escolas particulares, em cidades da região metropolitana de São Paulo, em espaço físico definido pelo responsável pelo local, onde os avaliados pudessem se sentir confortáveis ao realizar o teste. Foram incluídos todos aqueles que, atendendo aos critérios de inclusão, aceitaram participar voluntariamente do estudo.

A amostra foi de conveniência e o convite para participação ocorreu por meio de apresentação do projeto às escolas e consentimento dos responsáveis. Aos participantes foi apresentado o teste, dando-lhes liberdade para prosseguir ou não com as avaliações, que ocorreram durante o período de dois meses, sendo avaliados todos aqueles que manifestaram interesse na participação, até que fosse completado o número previsto de participantes.

A coleta de dados foi realizada em horário agendado com os participantes que aceitaram fazer parte do projeto, após a aprovação do estudo pelo Comitê de ética da Universidade. A aprovação foi registrada sob CAAE 45606715.4.0000.0084 e número de parecer 1.131.752. As avaliações ocorreram individualmente, em ambiente tranquilo, no qual estavam presentes o avaliador, um auxiliar e o avaliado.

Primeiramente foi aplicado o questionário de preferência manual de Van Strien ${ }^{11}$, o qual solicita ao avaliado a indicação da mão preferida para a realização de 11 tarefas: pegar no lápis quando desenha (1); segurar a escova (2); lavar os dentes (3); desenroscar a tampa de uma garrafa (4); lançar uma bola (5); dar as cartas de um baralho (6); pegar numa raquete (7); abrir a tampa de uma caixa (8); pegar numa colher quando come sopa (9); apagar com uma borracha (10); abrir uma porta com uma chave (11). Para a opção pela mão direita será atribuído o valor
+1, para a opção pela mão esquerda o valor -1 , e à opção por "qualquer delas", o valor 0. Os participantes foram classificados como fortemente destrímanos (com valores entre 8 e 10) e fortemente sinistromanos (com valores entre -10 e -8$)$.

Posteriormente os participantes foram convidados, sempre individualmente a realizarem o TCB. Para a aplicação do teste foram seguidas as recomendações descritas por Matchiowetz et al. ${ }^{3}$ e Mendes et al. ${ }^{4}$.

$\mathrm{O}$ ambiente de aplicação foi em um local sem ruídos e bem iluminado, com o participante sentado confortavelmente em uma cadeira adequada ao seu tamanho. A caixa com os blocos foi colocada à frente do aluno, em posição horizontal, com a divisória alinhada ao plano sagital da cabeça, permitindo assim total visão da área do equipamento usado para sua avaliação de destreza manual.

Todos os participantes puderam treinar por cerca de 15 segundos antes da aplicação do teste. Após o treino, os blocos foram colocados na posição inicial. Foram feitas duas avaliações sequenciais para a mão dominante, e duas para a mão não dominante, tomando-se a média dos blocos transferidos por minuto para os cálculos dos escores.

O tempo cronometrado foi de um minuto e em seguida o número de blocos transferidos foram computados, sendo este o escore do teste (blocos/minuto).

\section{RESULTADOS E DISCUSSÃO}

Observou-se interesse de todos os avaliados em realizar o teste e a todos foi dada uma devolutiva de desempenho, não havendo nenhuma recusa ou observação de dificuldade na compreensão da tarefa, que foi realizada por todos. Dentre os 120 participantes, 15 foram classificados como ambidestros pelo teste de preferência manual de Van Strien $^{11}$, sendo excluídos conforme os critérios propostos no método. Dentre os demais, 99 alunos eram destros (94,28\%) e seis eram canhotos $(5,72 \%)$. A amostra final do estudo foi composta por 105 crianças e adolescentes, divididos então em dois grupos, sendo Grupo 1: 7-10 anos, com 29 meninas e 26 meninos e Grupo 2: 11 a 14 anos, com 27 meninas e 23 meninos.

Os dados foram registrados em folhas individuais com os escores dos dois testes, Van Strien e TCB. Após a finalização da coleta foram tabulados em planilhas do Excel e separados por sexo e faixa etária para tratamento estatístico.

Os resultados sobre o desempenho no TCB foram organizados em tabelas considerando o sexo, a faixa etária do grupo, a mão dominante (MD) e mão não dominante (MND), com média das duas tentativas para os sujeitos, 
Turco BPBA, et al. Caracterização do desempenho de destreza manual. Rev Ter Ocup Univ São Paulo. 2018 maio-ago.;29(2):164-9.

desvio padrão e número de avaliados.

Foram realizados testes para normalidade da amostra (Anderson-Darling) e houve normalidade para os dois grupos. Foram realizados os testes Fisher para variâncias e t-student para as médias.

$\mathrm{Na}$ amostra estudada não houve diferença de desempenho entre o sexo feminino e masculino na faixa etária de 7-10 anos entre MD e MND. Já para o Grupo de 1114 anos, os resultados mostraram variâncias iguais e médias diferentes para MD e MND, sendo o desempenho melhor para o sexo feminino $(\mathrm{P}=0,000$ e $\mathrm{P}=0,005$, respectivamente).

Na comparação dos grupos por idade observou-se que para meninas e meninos, de ambas as faixas etárias e para MD e MND houve igualdade de variâncias. Porém, houve diferença de médias entre as faixas etárias para os grupos masculino e feminino, com melhor desempenho para o grupo de 11 a 14 anos, demonstrando que quando maior a idade, melhor o desempenho no teste para ambas as mãos com $\mathrm{p}=0,000$ para MD e MND para o sexo feminino e $\mathrm{p}=0,000$ e $\mathrm{p}=0,001$ para MD e MND no sexo masculino.

Os dados normativos da amostra em relação ao TCB encontram-se detalhados nas Tabelas 1 e 2, para meninas e meninos, organizadas com base no modelo de apresentação dos dados fornecidos por Mathiowetz et al. ${ }^{3}$ e por Mendes et al. ${ }^{4}$, que inclui idade, número de participantes (n), mão dominante e não dominante (MD e MND), média de cubos transportados por minuto e desvio padrão, número mínimo e máximo de cubos transportados.

Tabela 1: Dados normativos para o sexo feminino

\begin{tabular}{|c|c|c|c|c|c|c|}
\hline \multirow{2}{*}{ Idade (anos) } & N & Mão & Média & SD & Mínimo & Máximo \\
\hline \multirow{2}{*}{$7-10$} & 29 & MD & 62,03 & 5,816 & 46 & 91 \\
& \multirow{2}{*}{27} & MND & 60,07 & 7,987 & 40 & 91 \\
\hline \multirow{2}{*}{$\mathbf{1 1 - 1 4}$} & MD & 77,87 & 7,060 & 64 & 95 \\
& & MND & 73,32 & 8,552 & 56 & 90 \\
\hline
\end{tabular}

Tabela 2: Dados normativos para o sexo masculino

\begin{tabular}{|c|c|c|c|c|c|c|}
\hline \multirow{2}{*}{ Idade (anos) } & N & Mão & Média & SD & Mínimo & Máximo \\
\hline \multirow{2}{*}{$7-10$} & 26 & MD & 61,79 & 7,454 & 45 & 78 \\
& \multirow{2}{*}{23} & MND & 60,04 & 7,618 & 43 & 75 \\
\hline \multirow{2}{*}{$\mathbf{1 1 - 1 4}$} & & MD & 70,63 & 6,298 & 58 & 89 \\
& & MND & 66,78 & 6,770 & 55 & 88 \\
\hline
\end{tabular}

Neste estudo optou-se por avaliar e analisar os resultados considerando a preferência manual identificada pelo teste de Van Strien ${ }^{11}$, que determinou o percentual de destros e canhotos. Os estudos de Mathiowetz et al. ${ }^{3}$ e Mendes et al. ${ }^{4}$ fizeram a análise de desempenho considerando mão direita e esquerda, independente da preferência manual constatada. Considera-se, no entanto, de acordo com estudos mais atuais ${ }^{12}$ que a análise de assimetria manual ou preferência deve ser analisada de acordo com a mão dominante e não dominante, descartando-se assim, qualquer erro na interpretação dos dados.

Mathiowetz et al. ${ }^{3}$ não consideraram os ambidestros em sua avaliação de preferência manual, distribuindo os 628 participantes em destros e canhotos. A definição da lateralidade foi feita a partir de uma pergunta aos avaliados: você é destro ou canhoto? Os pesquisadores recrutaram os sujeitos em shoppings centers, feiras, centros de terceira idade, centros de reabilitação e universidade. O público de americanos participantes do estudo foi distribuído em faixas etárias agrupadas em 5 anos a partir dos 20 anos, até os 74, mais um grupo que reunia todos os participantes com mais de 75 anos. Cada grupo contava com no mínimo 21 e no máximo 29 integrantes para cada sexo. No presente estudo a amostra foi composta foi 105 participantes entre 7 e 14 anos, divididos em dois grupos (7-10 e 11-14), variando entre 23 e 29 participantes por sexo em cada uma das duas faixas etária agrupadas, seguindo distribuição semelhante ao estudo original de Mathiowetz. 
A validação do TCB no Brasil foi realizada com um grupo composto por 446 indivíduos sem disfunções ortopédicas ou neurológicas, na faixa compreendida entre 15 e 86 anos. Os autores encontraram 418 destros, 17 canhotos e 11 ambidestros. O percentual de indivíduos canhotos e ambidestros foi de aproximadamente $6 \%$, enquanto que no presente estudo foi de $17,5 \%$ considerando também os canhotos e os ambidestros ${ }^{4}$. Essa diferença pode estar ligada à idade investigada, uma vez que existe grande percentual de ocorrência de lateralidade cruzada em escolares nos primeiros anos do ensino fundamental ${ }^{13}$.

$\mathrm{O}$ estudo de Mendes et al. ${ }^{4}$ agrupou as faixas etárias em blocos de dez anos $(15-24 ; 25-34$; 35-44; e 45 54 anos, com o último grupo sendo composto por sujeitos acima dos 55 anos) $\mathrm{O}$ número de integrantes por grupo, também separados por homens e mulheres, variou de 38 a 65 . Deve-se considerar que o presente estudo avaliou faixas etárias com menos de 5 anos de diferença (7-10 e 11-14), complementando os grupos não contemplados ainda pelos parâmetros do TCB por Mathiowetz et al. ${ }^{3}$ e Mendes et al. ${ }^{4}$.

No Brasil, foi encontrado um estudo que teve como foco o uso do TCB com crianças e jovens com síndrome de Down (SD) e que, para efeitos comparativos, realizou o teste em um grupo controle (GC) nas faixas etária entre 7 e 9 anos e 14 e 15 anos, com o propósito de comparar a evolução da destreza manual de acordo com o aumento da idade. Esta hipótese foi confirmada para o $\mathrm{GC}$, mas não para o grupo com SD, para o qual não houve mudanças de desempenho com o passar do tempo [5]. A média de blocos transportados para GC, que foi composto por crianças e adolescentes de 7 , $8,9,14$ e 15 anos ficou entre 60 e 79 para MD e 58 e 75 para $\mathrm{MND}$, sem diferenciação entre os sexos. Porém não foram coletados os dados com as idades de 10 a 13 anos. Pode-se observar que a média de blocos por minuto transferidos pela mão dominante e não dominante não se diferenciam muito dos resultados obtidos neste estudo, encontrando-se em ambos os grupos a melhora do desempenho manual de acordo com o avanço da idade.

Os dados aqui discutidos demonstram a facilidade da aplicação do teste, com boa compreensão entre os participantes e bom desempenho, tornando-o um instrumento eficaz para a avaliação do desempenho manual devida à sua prática confecção e baixo custo. As medidas e instruções para a obtenção do material encontramse disponíveis no artigo original ${ }^{3}$, bem como todas as instruções para aplicação, que foram publicadas também pelo grupo de Mendes et al. ${ }^{4}$, com objetivo de validar o teste para a população brasileira e utilizá-lo também com pacientes com esclerose múltipla.

Foram encontrados até abril de 2018, nas bases de dados Lilacs, Medline, Pubmed, Web of Science e SciELO, 12 trabalhos realizados no Brasil que fizeram uso do TCB, todos após a publicação de Mendes et al. ${ }^{4} \mathrm{em}$ 2001. Os trabalhos estão concentrados entre 2009 e 2016, com populações com acidente vascular encefálico (6); esclerose múltipla (2) e síndrome de Down (4). Observa-se um predomínio de público adulto nas pesquisas, voltadas em sua maioria para pacientes com acidente vascular encefálico, com foco na mão espástica ${ }^{14,15,16,17,18,19}$ e esclerose múltipla ${ }^{20,21}$. Os estudos com a população infantil ou de adolescentes ocorreu apenas com a síndrome de Down e estão disponíveis em menor número nas bases de dados consultadas ${ }^{1,6,22,23}$. A criação de parâmetros para faixas etárias mais jovens abre caminhos para a avaliação da destreza para este público, cujo foco na atividade manual pode beneficiar as atividades escolares e de vida diária.

Com o uso de avaliações objetivas torna-se possível as ações diagnósticas e interventivas que fundamentem uma prática baseada em evidências na área da reabilitação.

\section{CONSIDERAÇÕES FINAIS}

Os dados aqui apresentados permitiram a criação de parâmetros para a faixa etária entre 7 e 14 anos, complementando os escores já encontrados na literatura que se concentravam em idades a partir dos 15 anos. O uso do TCB é simples e adequado para faixas etárias mais jovens que as até então descritas, abrindo possibilidades para maior precisão na avaliação da função manual e possibilitando melhor planejamento terapêutico para as diversas faixas etárias e diferentes quadros clínicos.

Participação dos autores no texto: Turco BPBA: Responsável pela elaboração do projeto de pesquisa, coleta de dados em campo e redação de relatório final; Cymrot R: Responsável pela definição de método de análise dos dados, estatística e discussão dos resultados, Blascovi-Assis SM: Responsável pela definição de tema, orientação do trabalho, elaboração do projeto, supervisão de coleta, discussão e revisão final do trabalho. 
Turco BPBA, et al. Caracterização do desempenho de destreza manual. Rev Ter Ocup Univ São Paulo. 2018 maio-ago.;29(2):164-9.

\section{REFERÊNCIAS}

1. Souza A, Cymrot R, Vianna D, Caromano F, Blascovi-Assis SM. Síndrome de Down: correlação entre o desempenho funcional com a força de preensão palmar e a destreza manual. Fisioter Bras. 2012;13(3):211-5.

2. Magill RA. Aprendizagem motora: conceitos e aplicações. São Paulo: Editor Edgard Blucher; 2000.

3. Mathiowetz V, Volland G, Kashman N, Weber K. Adult Norms for the Box and Block Test of Manual Dexterity. Am J Occup Ther. 1985;39(6):386-91 doi: 10.5014/ajot.39.6.386.

4. Mendes MF, Tilbery CP, Moreira MA, Cruz AMB. Teste de destreza manual da caixa em blocos em indivíduos normais e em pacientes com esclerose múltipla. Arq Neuropsiquiatria. 2001;59(4):889-94. doi:10.1590/S0004-282X2001000600010.

5. Guimarães R, Blascovi-Assis SM. Uso do teste caixa e blocos na avaliação de destreza manual em crianças e jovens com síndrome de Down. Rev Ter Ocup Univ São Paulo. 2012;23(1):98-106.

6. Priosti PA, Blascovi-Assis SM, Cymrot R, Vianna LD, Coromano FA. Força de preensão e destreza manual na criança com Síndrome de Down. Fisioter. Pesqui; 2013;20(3):278-85. doi: 10.1590/S1809-29502013000300013.

7. Freitas C, Vasconcelos O, Botelho M. Lateralidade e coordenação motora em crianças dos 4 aos 6 anos. Um estudo com o teste M-ABC. In: Morouço P, Vasconcelos O, Barreiros J, Matos R, editores. Estudos em desenvolvimento motor da criança IV. Leiria: Instituto Politécnico, Escola de Educação; 2011. p.111-7.

8. Coppede AC, Campos AC, Santos DCC, Rocha NACF. Desempenho motor fino e funcionalidade em crianças com síndrome de Down. Fisioter Pesqui. 2012;19(4):363-8. doi: 10.1590/S1809-29502012000400012.

9. Chultes L, Valentini NC. Alfabetização e desenvolvimento motor: um estudo sobre o desenvolvimento da escrita e da motricidade fina de crianças dos $1^{\circ}$ e $2^{\circ}$ anos do ensino fundamental. Temas Desenvolv. 2014;20(109):56-62. Disponível em: https://tinyurl.com/ycx7fur6.

10. Magalhães LC, Rezende MB, Cardoso AM, Galvão BAP, Maor FMO. Relação entre destreza manual e legibilidade da escrita em crianças: estudo piloto. Rev Ter Ocup Univ São Paulo. 2011;22(2):127-35. doi: https://doi.org/10.11606/ issn.2238-6149.v22i2p127-135.

11. Van Strien JW. Classificatie van links: en rechtshangige proefperrsonen [Classification of left-and right-handed research participants]. Nederlands Tijdschrift voor de Psychologie, Amsterdam. 1992;47:88-92. Available from: https://tinyurl.com/yan2v2gd.

12. Freitas C, Botelho M., Vasconcelos O. Preferência lateral e coordenação motora. Motricidade. 2014;10(2):11-24. doi: 10.6063/motricidade.10(2).1245.

Recebido em: 08/05/2018

Aceito em: 18/06/2018
13. Rosa Neto F, Xavier RFC, Santos APM, Amaro KN, Florêncio R, Poeta LS. A lateralidade cruzada e o desempenho da leitura e escrita em escolares. Rev CEFAC. 2013;15(4):864-72. http:// dx.doi.org/10.1590/S1516-18462013000400015.

14. Soares AV, Woellner SS, Andrade CS, Mesadri TJ, Bruckheimer AD, Hounsell MS. The use of Virtual Reality for upper limb rehabilitation of hemiparetic Stroke patients. Fisioter Mov. 2014;27(3):309-17. doi: 10.1590/0103-5150.027.003.AO01.

15. Araújo M, Postól MK, Bruckheimer AD, Hounsell MS, Woelner SS, Soares AV. Realidade virtual: efeitos na recuperação do membro superior de pacientes hemiparéticos por acidente vascular cerebral. Arq Catarin Med. 2014;43(1):1520. Disponível em: http://www.acm.org.br/revista/pdf/ artigos/1267.pdf.

16. Woellner SS, Soares AV, Cremonini CR, Cremonini L, Domenech SC, Borges Júnior, NG. Treinamento específico do membro superior de hemiparéticos por acidente vascular encefálico. Arq Catarin Med. 2012;41(3):49-53. Disponível em: http://www.acm.org.br/revista/pdf/artigos/944.pdf.

17. Soares AV, Kerscher C, Uhlig L, Domenech SC, Borges Júnior, NG. Dinamometria de preensão manual como parâmetro de avaliação funcional do membro superior de pacientes hemiparéticos por acidente vascular cerebral. Fisioter Pesqui. 2011;18(4):359-64. doi: 10.1590/S1809-29502011000400011.

18. Soares VA, Kerscher C, Uhlig L. Domenech CS, Borges Júnior, NG. Escala de movimentos da mão: um instrumento preditivo da recuperação funcional do membro superior de pacientes hemiparéticos por acidente vascular cerebral. Arq Catarin Med. 2011;40(2):47-51. http://www.acm.org.br/revista/pdf/ artigos/944.pdf

19. Garros DSC, Gagliardi RJ, Guzzo RAR. Evaluation of performance and personal satisfaction of the patient with spastic hand after using a volar dorsal orthosis. Arq Neuro-Psiquiatr. 2010;68(3):385-9. doi: 10.1590/S0004282X2010000300011.

20. Nascimento MM, Ribeiro SBF, Barroso SM, Silva C, Ribeiro JB, Cardoso, Fabrizio AG. Força de pinça trípode e destreza manual em pacientes portadores de esclerose múltipla forma remitente-recorrente. Fisioter Bras, 2013;14(4):283-8.

21. Nogueira LAC, Nóbrega FR, Lopes KN, Thuler LCS, Alvarenga RMP. The effect of functional limitations and fatigue on the quality of life in people with multiple sclerosis. Arq Neuro-Psiquiatr. 2009;67(3b):812-7. doi:10.1590/S0004282X2009000500006.

22. Rufino LA, Blascovi-Assis SM, Souza AB, Verginassi G, Cymrot R. Avaliação da destreza manual em pessoas com síndrome de Down: comparação entre teste caixa e blocos, Minnesota e Jebsen-Taylor. Fisioter Bras. 2016;17(3):188-96.

23. Guimarães R, Blascovi-Assis SM, Macedo EC. Efeito da dominância lateral no desempenho da destreza manual em pessoas com síndrome de Down. Acta Fisiatr. 2012,19(1):6-10. doi: https://doi.org/10.5935/0104-7795.20120002. 